\title{
Improving Oxygen Binding of Desiccated Human Red Blood Cells
}

\author{
Steingrimur Stefansson1, David S. Chung2, Jamie Yoon ${ }^{3}$, Won Seok Yoo4, \\ Young Wook Park ${ }^{5}$, George Kim ${ }^{6}$, David Hahn', Huyen Le' ${ }^{7}$, Sung-Jae Chung, ${ }^{6,8}$, \\ Stephen P. Bruttig' ${ }^{1}$ David H. Ho ${ }^{*}$ \\ ${ }^{1}$ HeMemics Biotechnologies Inc., Rockville, MD, USA \\ ${ }^{2}$ Division of Biology \& Medicine, Brown University, Providence, RI, USA \\ ${ }^{3}$ Earl Warren College, UC San Diego, CA, USA \\ ${ }^{4}$ Department of Chemistry, Michigan University, Ann Arbor, MI, USA \\ ${ }^{5}$ Seoul International School, Seongnam, South Korea \\ ${ }^{6}$ Fuzbien Technology Institute, Rockville, MD, USA \\ ${ }^{7}$ Nauah Solutions, Mclean, VA, USA \\ ${ }^{8}$ Marymount University School of Arts \& Science, Arlington, VA, USA \\ Email: *dho@hememics.com
}

Received 4 January 2016; accepted 1 February 2016; published 4 February 2016

Copyright (C) 2016 by authors and Scientific Research Publishing Inc.

This work is licensed under the Creative Commons Attribution International License (CC BY). http://creativecommons.org/licenses/by/4.0/

(c) (i) Open Access

\begin{abstract}
Desiccating human red blood cells (RBCs) to increase their storage life has been the subject of intense research for a number of years. However, drying RBCs invariably compromises their integrity and has detrimental effects on hemoglobin function due to autoxidation. We have previously demonstrated an RBC desiccation and rehydration process that preserves RBC antigenic epitopes better than frozen RBCs. This study expands on those observations by examining what effects this desiccation process has on RBC hemoglobin function with respect to oxygen binding properties. In this paper, we examined RBCs from normal donors which were desiccated to $25 \%$ moisture content and stored dry for 2 weeks at room temperature prior to rehydration with plasma followed by structural and functional studies. Our data showed that approximately $98 \%$ of the RBCs were intact upon rehydration based on hemolysis assays. Oxygen dissociation curves for the desiccated/rehydrated RBCs showed a left shift compared to fresh $\mathrm{RBCs}\left(\mathrm{pO}_{2}=17 \mathrm{mmHg}\right.$ vs. $26 \mathrm{mmHg}$, respectively). The desiccated/rehydrated RBCs also showed an increase in methemoglobin compared to fresh RBCs (4.5\% vs $0.9 \%$, respectively). 2,3-Diphosphoglycerate concentration of the desiccated/rehydrated RBCs was reduced by $20 \%$. In conclusion, although this RBC dehydration process preserves RBC integrity and hemoglobin oxygen binding properties better than most other dehydration techniques described so far, further optimization and long-term studies are needed to make this procedure acceptable for human transfusion.
\end{abstract}

"Corresponding author.

How to cite this paper: Stefansson, S., Chung, D.S., Yoon, J., Yoo, W.S., Park, Y.W., Kim, G., Hahn, D., Le, H., Chung, S.-J., Bruttig, S.P. and Ho, D.H. (2016) Improving Oxygen Binding of Desiccated Human Red Blood Cells. Advances in Bioscience and Biotechnology, 7, 47-54. http://dx.doi.org/10.4236/abb.2016.72006 
Keywords

Desiccation, Red Blood Cells, Oxygen Binding, Hemolysis, Methemoglobin, 2,3-Diphosphoglycerate

\section{Introduction}

Stabilization of RBCs that allows for dried storage at room temperature, without causing prohibitive hemolysis and hemoglobin oxidation, could potentially change the future of blood banking because it offers numerous advantages over $4^{\circ} \mathrm{C}$ and frozen storage. Dry storage that could extend the product's shelf life, will ease logistical problems, facilitate efficient transportation and save energy by eliminating the need for refrigeration [1].

Currently, the only viable alternative to storing RBCs at $4^{\circ} \mathrm{C}$ is freezing. However, freezing cells in the absence of cryoprotective agents cause irreparable cellular damage because ice crystals puncture membranes and organelles [2]. Apart from the physical damage, ice crystals inflict on cellular structures; the freeze-drying process itself can damage and/or oxidize proteins, including hemoglobin ( $\mathrm{Hb})$ [1]. Hb inside RBCs is also vulnerable to oxidation in the freeze-drying process. Oxidized $\mathrm{Hb}$ (met-hemoglobin) does not bind oxygen [3]. The oxidation of $\mathrm{Hb}$ within the RBCs can also lead to formation of intracellular reactive oxygen species that can damage lipids and structural proteins [4]. Attempts at freeze-drying RBCs have mostly resulted in poor cellular recovery [1] [2] [4].

Many factors can influence recovery of functional RBCs after freeze-drying. This includes addition of cryoprotectants (e.g. glycerol and DMSO), lyoprotectants (e.g. trehalose and polymers), manipulating the operating condition during the drying process (primary and secondary drying) and the rehydration process. As of yet, recovery of intact RBCs after drying and rehydration has been reported to be between $50 \%$ and $85 \%$ [5]-[8], but because of elevated $\mathrm{Hb}$ oxidation, no RBC desiccation procedures meet the standards for transfusion at present [1] [4].

Here, we present a storage process for RBCs that utilizes desiccation. The desiccation process does not involve freezing; thus ice-crystal damage and hemolysis is avoided. The overall desiccation/rehydration process involves several steps including incubation in HemSol ${ }^{\mathrm{TM}}$ which stabilizes the RBC morphology prior to desiccation. HemSol ${ }^{\mathrm{TM}}$ contains a proprietary mixture of non-toxic high and low molecular weight carbohydrates, including trehalose, mannose and dextrans that protect RBC integrity and protein function during the desiccation and rehydration process. Previously we demonstrated that RBCs desiccated and stored in HemSol ${ }^{\mathrm{TM}}$ for over 3 weeks at room temperature can be used, in lieu of fresh RBCs, as standards for hemagglutination assays [8].

This RBC desiccation process did not involve freezing. HemSol ${ }^{\mathrm{TM}}$ treated RBCs were dried in a vacuum oven at low temperature $\left(42^{\circ} \mathrm{C}\right)$ until approximately $25 \%$ of the water was remaining. That study showed that our HemSol ${ }^{\text {TM }}$ desiccation process preserves RBC morphology and blood typing epitopes better than RBCs that had been frozen. This opens up the possibility of using desiccated RBCs to archive serological phenotypes for use in reference laboratories.

In this study we expand on these previous results by examining whether HemSol ${ }^{\mathrm{TM}}$ treatment alters the main physiological property of RBCs, namely the oxygen carrying capacity of Hb. RBCs used in this study were stored desiccated in HemSol ${ }^{\mathrm{TM}}$ at room temperature for 2 weeks prior to being rehydrated. Our results demonstrate that the desiccated/rehydrated cells have functional Hb. Compared to fresh RBCs, desiccated and rehydrated RBCs were $98 \% \pm 1 \%$ intact based on hemolysis and could be used for these assays directly without a washing step. The rehydrated RBCs demonstrated a left shift in the $\mathrm{pO}_{2}$ (p50) oxygen saturation value compared to fresh control RBCs (17.10 mmHg vs. $26.48 \mathrm{mmHg}$, respectively). The reasons for this left shift could be due to increased methemoglobin levels (4.5\%) and/or decreased 2,3-diphosphoglycerate (DPG) levels (20\%).

To our knowledge, this is the first time these types of experiments have been performed on Hb function in RBC that have been desiccated for 2 weeks at RT. This is a promising start for developing a procedure for producing desiccated RBCs suitable for human transfusion, but further studies are needed to optimize this process.

\section{Materials and Methods}

Red Blood Cells and Morphology: In-dated human blood, no older than 1 - 3 day was obtained from the Red Cross (American Red Cross, Rockville, MD) as packed red cells. Cells were checked for count per unit volume, 
morphology, hemolysis and crenated cells. Evaluations were performed according to standard manual methods for counting (hemocytometer), microscopy and digital photo-microscopy. For morphology experiments, the RBCs were divided into 2 equal parts, one part to be used for desiccation process and room temperature storage and the other part was kept refrigerated at $4^{\circ} \mathrm{C}$ as a control.

Aliquots of the reconstituted RBCs were diluted 1:10,000 in saline and examined for morphology under a compound microscope at 400× magnification. For each sample, 5 independent fields each containing at least 100 RBCs were examined.

Desiccation and Rehydration of Red Blood Cells: RBCs were mixed with HeMemics' desiccation buffer called HemSol ${ }^{\mathrm{TM}}$ as described previously [8]. Briefly, a HEPES buffer $\mathrm{pH} 7.4$ contained a proprietary ratio of low molecular weight sugars (Trehalose, Mannitol and Glucose) and high molecular weight sugar Dextran. For these experiments, RBCs were washed with saline 3 times using low speed centrifugation $(100 \times \mathrm{g}$ for $10 \mathrm{mi}-$ nutes) to remove residual plasma. The packed RBCs were then mixed with HemSolTM at 1:1 ration and allowed to equilibrate at room temperature for 30 minutes. Then, $0.5 \mathrm{~mL}$ volumes of the red blood cells mixed with HemSol $^{\mathrm{TM}}$ were dried in $20 \mathrm{~mL}$ screw top glass vials. Specifically, aliquots of the blood-desiccation buffer mix which were dispensed into glass vials were loaded into a vacuum drying oven and dehydrated under mild heat $\left(42^{\circ} \mathrm{C}\right)$ until visibly dry. The percent moisture of the resulting RBC preparations was determined by gravimetric drying method using the Sartorius MA45 Gravimetric Moisture Balance (PWB, Bradford, MA). The samples were then capped and stored at room temperature $\left(23^{\circ} \mathrm{C}-25^{\circ} \mathrm{C}\right)$ until use.

For the rehydration process, the desiccated cells were rehydrated in human plasma. Plasma $(1 \mathrm{~mL})$ was added to the cells in the vial and allowed to incubate for 30 minutes at $37^{\circ} \mathrm{C}$ with gentle swirling or vial rotation to insure uniform rehydration. To ensure sufficient volume for testing once rehydration was completed, contents of several vials were combined and adjusted to $45 \%$ hematocrit with human plasma for testing.

Post-Rehydration Evaluation for Hb-Oxygen Dissociation Curve Analysis: After 2 weeks of storage in HemSol ${ }^{\mathrm{TM}}$ at RT, the desiccated blood was reconstituted as described above and oxygen dissociation curve testing was conducted. The Hemox-Analyzer (TSC Scientific Corporation, New Hope, PA) was used to determine the oxygen dissociation curve (ODC) by exposing $50 \mu \mathrm{L}$ of blood to an increasing partial pressure of oxygen and deoxygenating it with nitrogen gas. A Clark oxygen electrode was used to detect the change in oxygen tension, which was recorded with on the $x$-axis of an $x$-y recorder. The resulting increase in oxyhemoglobin fraction was monitored simultaneously by dual wavelength spectrophotometry at $560 \mathrm{~nm}$ and $576 \mathrm{~nm}$ and displayed on the y-axis. For the assays, reconstituted RBC (45\% HCT) prepared in plasma with heparin anticoagulant was kept on wet ice until the assay. Fifty $\mu \mathrm{L}$ of $\mathrm{RBC}$ was diluted in $5 \mu \mathrm{L}$ of Hemox buffer $(7.4 \pm 0.01)$. The sample-buffer was drawn into a cuvette and the temperature of the mixture was equilibrated and brought to $37^{\circ} \mathrm{C}$; the sample was oxygenated to $100 \%$ with air. After adjustment of the $\mathrm{pO}_{2}$ value, the sample was deoxygenated with nitrogen; during the deoxygenation process the curve was recorded on graph paper. The $\mathrm{pO}_{2}$ (p50) value was extrapolated on the x-axis as the point at which $\mathrm{O}_{2}$ saturation was 50\%. Two different lots of desiccated RBCs were tested and from each lot, samples were tested in triplicate.

Determination of Hemolysis: A sample of control and reconstituted RBCs were centrifuged at $3000 \times \mathrm{g}$ for 10 min to pellet the cells. Absorbance of the supernatant was measured at $450 \mathrm{~nm}$ and compared to the absorbance of the lysed pellet to give \% hemolysis.

Met-Hb Determination: To lyse RBC, $0.5 \mathrm{~mL}$ of desiccated/reconstituted RBC at $45 \%$ hematocrit was washed in saline 3 times via centrifugation at $500 \mathrm{~g}$ for 15 minutes. To the RBC pellet, $5.5 \mathrm{~mL}$ of distilled water was added and allowed to stand for $5 \mathrm{~min}$ at RT. The samples was diluted with $4 \mathrm{~mL}$ of $0.1 \mathrm{M}$ PBS, pH 6.8, mixed well and centrifuged at $3000 \times \mathrm{g}$ for $10 \mathrm{~min}$. The supernatant was removed for Met $\mathrm{Hb}$ analysis. Briefly, $0.5 \mathrm{~mL}$ of $4 \%$ (w/vol) $\mathrm{K}_{3} \mathrm{Fe}(\mathrm{CN})_{6}$ was added to $3 \mathrm{~mL} \mathrm{RBC}$ supernatant and allowed to sit for $10 \mathrm{~min}$, and $\mathrm{A} 630 \mathrm{~nm}$ measured using a spectrophotometer. To calculate baseline, $0.5 \mathrm{~mL}$ water was added to a fresh $3 \mathrm{~mL}$ sample of RBC supernatant and absorbance was measured at $630 \mathrm{~nm}$. The reaction was then neutralized by the addition of $0.050 \mathrm{~mL} 5 \%(\mathrm{w} / \mathrm{vol}) \mathrm{KCN}$ and after $5 \mathrm{~min}$. absorbance $630 \mathrm{~nm}$ was measured again. Met-Hb was calculated as the ratio of the absorbance change at $630 \mathrm{~nm}$ after adding KCN to the RBC supernatant. Control RBC sample was treated in the same manner to extract Met-Hb for measurements. All samples were tested in triplicate.

Measurement of 2,3-DPG (DPG): The assay was performed on 2 week old RBC samples stored desiccated in HemSol $^{\mathrm{TM}}$ and fresh RBCs using a commercially available human DPG ELISA Kit (antibodiesonline.com, Atlanta, GA). The microtiter plate provided in this kit had been pre-coated with an antibody specific to DPG. Procedures were well described in the protocol provided with the kit. Briefly, the RBC supernatant prepared as de- 
scribed in the Met-Hb determination section was added to the appropriate microtiter plate wells with a biotin-conjugated polyclonal antibody preparation specific for DPG. Avidin conjugated to Horseradish Peroxidase (HRP) was added to each well and incubated, followed by addition of TMB substrate solution. The peroxidase reaction was terminated by the addition of a sulphuric acid solution, and A450 was measured in a spectrophotometer. The concentration of DPG in the samples was calculated from a DPG standard curve. Control RBC sample was treated in the same manner to extract Met-Hb for measurements. All samples were tested in triplicate.

\section{Results}

Red blood cells were desiccated to different levels of dryness and then the percentage of overall hemolysis was determined. Samples were reconstituted with plasma and assayed for hemolysis as described. Table 1 shows that removal of more than $25 \%$ of residual moisture resulted in an increase in cell fragility upon reconstitution with increased time of storage. Thus, based on these observations, the subsequent data were performed on RBC at $25 \%$ residual moisture at 2 week storage cut off.

Changes in RBC morphology after the desiccation/rehydration process were evaluated by gross microscopic examination. Typical, healthy red blood cells are round, smooth bi-concave disks with a pink or red color. Other forms of RBCs, such as crenated (partially shrunken) and spherocytes can be present [9]. Crenated RBCs are associated with storage lesion, but also occur in response to high osmotic conditions or drying. Crenation is often reversible and while exposure to the high osmotic load of HemSol ${ }^{\mathrm{TM}}$ does cause some crenation, with proper rehydration, most of the crenated cells revert to smooth bi-concave disks after $30 \mathrm{~min}$ in human plasma (Figure 1(a)). Figure 1(b) shows control RBCs kept for 2 weeks at $4^{\circ} \mathrm{C}$.

The critical physiologic parameter for RBC preparations is their ability to properly bind, carry and release oxygen. The determinations of this ability are conducted by exposing the RBCs to oxygen to assure complete loading, and then exposure to complete nitrogen to determine the continuous unloading of the oxygen (oxyhemoglobin dissociation curve). Typical oxyhemoglobin dissociation curve determinations are presented in Figure 2 and demonstrate a sigmoidal curve for both the fresh control RBCs and the desiccated/rehydrated RBCs, indicating cooperative oxygen binding.

Met-Hb is a measure of hemoglobin oxidation and is expressed as a proportion of total hemoglobin. Met-Hb is a naturally occurring oxidized metabolite of hemoglobin; under normal physiologic conditions, or in fresh RBCs, around $1 \%$ of total $\mathrm{Hb}$ is Met-Hb [10]. In desiccated/reconstituted RBCs, Met-Hb levels were $4.56 \% \pm$ 0.15\% upon reconstitution. Fresh control RBCs showed 0.9\% \pm 0.12\% Met-Hb (Figure 3(a)).

\section{Table 1. Correlation of RBC hemolysis to moisture content.}

\begin{tabular}{ccccc}
\hline \% Residual Moisture & \% Hemolysis at Day 1 & \% Hemolysis at Day 7 & \% Hemolysis at Day 14 & \% Hemolysis at Day 21 \\
\hline $10 \pm 0.8$ & $9.5 \pm 1.1$ & $11.4 \pm 1.4$ & $11.8 \pm 2.2$ & $15.5 \pm 2.4$ \\
$15 \pm 0.7$ & $5.5 \pm 1.3$ & $6.1 \pm 1.1$ & $7.3 \pm 1.8$ & $10.1 \pm 2.1$ \\
$20 \pm 0.8$ & $2.0 \pm 0.7$ & $2.5 \pm 0.6$ & $3.0 \pm 0.8$ & $5.5 \pm 1.2$ \\
$25 \pm 1.0$ & $0.4 \pm 0.2$ & $0.5 \pm 0.1$ & $0.6 \pm 0.3$ & $1.5 \pm 0.5$ \\
$30 \pm 1.2$ & $0.3 \pm 0.2$ & $0.4 \pm 0.1$ & $0.7 \pm 0.2$ & $1.8 \pm 0.5$ \\
$35 \pm 1.3$ & $0.5 \pm 0.1$ & $0.4 \pm 0.3$ & $0.4 \pm 0.2$ & $0.9 \pm 0.3$ \\
$40 \pm 1.3$ & $0.3 \pm 0.2$ & $0.5 \pm 0.4$ & $0.7 \pm 0.3$ & $0.8 \pm 0.2$ \\
$45 \pm 1.2$ & $0.5 \pm 0.3$ & $0.6 \pm 0.2$ & $0.5 \pm 0.3$ & $1.0 \pm 0.2$ \\
$50 \pm 1.1$ & $0.2 \pm 0.1$ & $0.5 \pm 0.2$ & $0.7 \pm 0.3$ & $0.8 \pm 0.2$ \\
$55 \pm 1.3$ & $0.5 \pm 0.3$ & $0.4 \pm 0.1$ & $0.6 \pm 0.2$ & $0.8 \pm 0.4$ \\
Control RBC & $0.4 \pm 0.2$ & $0.3 \pm 0.1$ & $0.4 \pm 0.1$ & $0.6 \pm 0.1$ \\
\hline
\end{tabular}

Data from 5 lots of RBC purchased commercially. Each samples were tested in triplicate \pm Standard deviation. Samples were pooled from storage and tested at time intervals indicated. Control RBC samples were stored refrigerated in accordance with FDA requirements. 


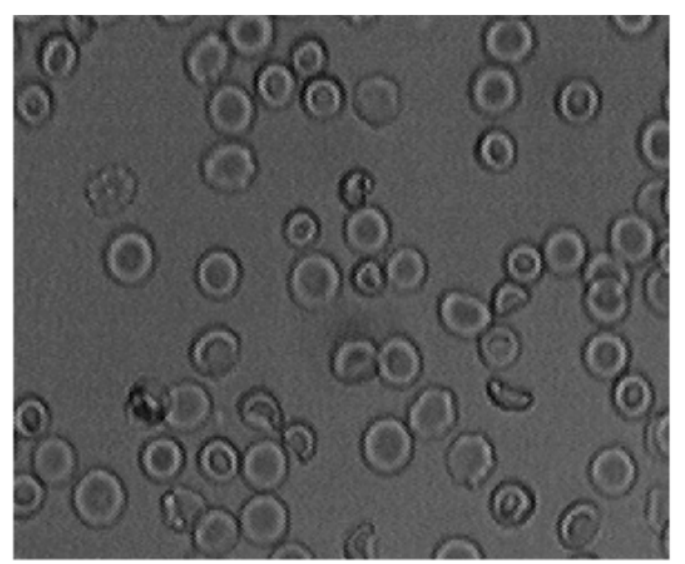

(a)

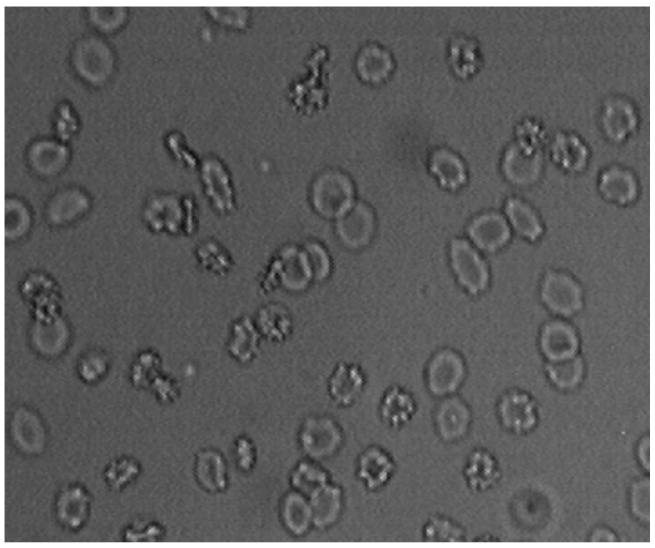

(b)

Figure 1. Light field images of RBCs. (a) RBCs stored desiccated for 2 weeks and reconstituted in human plasma and (b) RBCs stored for 2 weeks at $4^{\circ} \mathrm{C}(400 \times$ magnification).

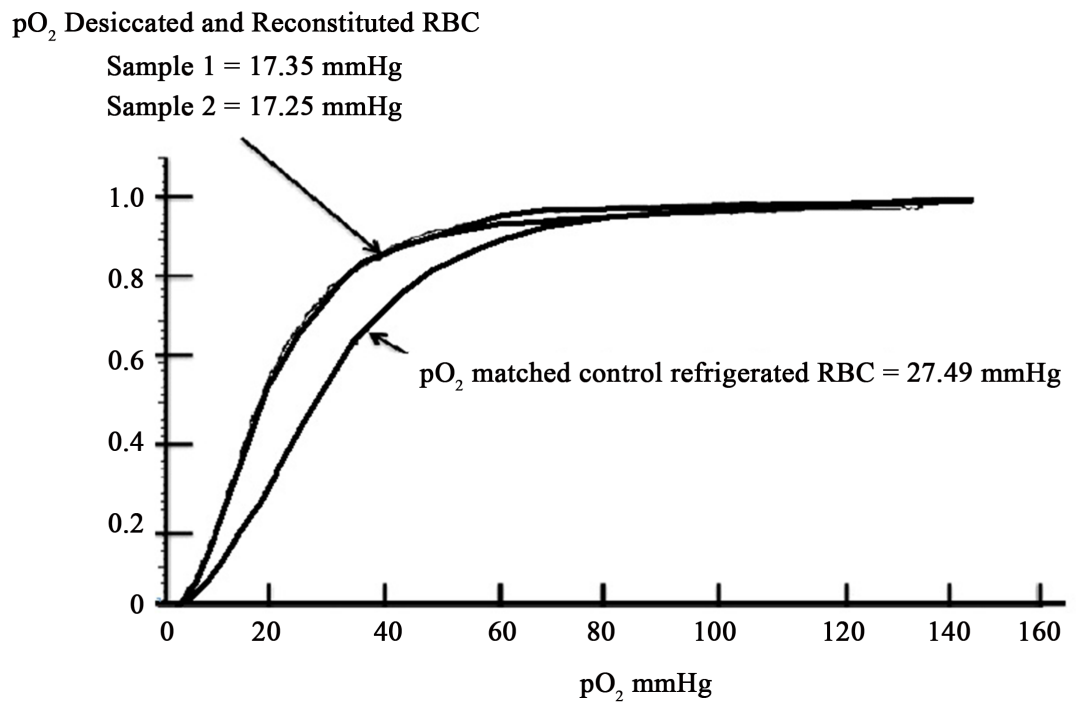

Figure 2. Oxygen binding and release profile for reconstituted RBCs. Dried RBCs were prepared in two separate sets from two different donors and kept for 2 weeks at room temperature before testing. The fresh and rehydrated RBCs (from both sets) showed similar oxygen saturation kinetics (99.4\%). The P50 of the rehydrated RBCs (17.35 for set 1 and 17.25 for set 2 ) were somewhat reduced compared to the fresh RBC $(\mathrm{P} 50=27.49)$. These assays were performed in triplicate.

\section{Desiccated RBC}

\section{Control RBC}

0

$\begin{array}{cccc}0 & 2 & 4 & 6\end{array}$

(a)

\section{Desiccated RBC}

\section{Control RBC}

$\begin{array}{llrr}0 & 50 & 100 & 150 \\ & \% & 2,3-\mathrm{DPG}\end{array}$

(b)

Figure 3. Measurements of Met Hb and DPG levels in RBC. (a) Met-Hb content increased in desiccated RBC compared to fresh RBC; (b) The assay for DPG content showed a decrease of approximately $20 \%$ for desiccated RBCs upon reconstitution compared to fresh RBCs. These assays were performed in triplicate. 
2,3-Diphosphoglycerate (DPG) is an important regulator of Hb oxygen affinity and we measured DPG concentrations in desiccated/rehydrated RBCs stored for 2 weeks at room temperature in HemSol ${ }^{\mathrm{TM}}$. Using fresh RBCs as control, we found that the concentrations of DPG in the desiccated/rehydrated RBCs were reduced by $20 \%$ (Figure 3(b)).

\section{Discussion}

RBCs destined for transfusion must be stored refrigerated at $4^{\circ} \mathrm{C}$ with a maximum allowable storage time of 42 days [9] [11]. Freezing RBCs and storing them in frozen state is an alternative to cold storage. Subzero temperatures decrease most biochemical reactions and frozen RBCs have been shown to have a longer shelf life [12]. However, the cryopreservation technique has disadvantages because of the need to introduce sometimes toxic antifreezing agents (e.g. DMSO, high concentrations of glycerol) into the blood before the freezing process and removing them before transfusion, all the while maintaining sterility. Because of the labor-intensive and technically demanding nature of processing coupled with the high cost, frozen RBCs are not in routine clinical use. Furthermore, freezing RBCs, even in the presence of cryoprotective agents does not eliminate hemolysis or hemoglobin oxidation. The damage caused by freezing and thawing also leaves behind cellular debris and materials unacceptable for transfusion [1] [13].

Drying RBCs in the absence of freezing step and keeping them functional is even more challenging than freeze-drying [13]. Most biochemistry occurs in an aqueous environment but a few organisms can survive extensive dehydration, a process termed anhydrobiosis. Drying cells without destroying them requires the presence of lyoprotectants. The most common lyoprotectant found in nature is the disaccharide trehalose, although trehalose alone is not sufficient to ensure full integrity upon rehydration [14].

The HemSol ${ }^{\mathrm{TM}}$ formulation used in this study contains trehalose along with other carbohydrates that have been shown to be non-toxic in humans. This RBC drying process does not involve freezing and has been shown to preserve RBC integrity and antigenicity better than freezing [8]. For this study we used an improved HemSol ${ }^{\mathrm{TM}}$ formulation that recovered $98 \% \pm 1 \%$ of the RBCs after reconstitution compared to $50 \%$ as we reported previously [8]. In contrast, most studies on drying and reconstituting RBCs have reported recoveries between $20 \%-50 \%[5]-[8]$.

Before assessing the oxygen binding properties of RBC, we performed experiments to determine the optimal moisture content and storage time for the desiccated RBC. Our data showed that the $25 \%$ residual moisture was the optimal level of dryness for RBC using our process. This data also suggested that removal of more than $75 \%$ of cell water content may compromise cell membrane structure that could cause higher breakage upon reconstitution. Furthermore, we also showed that beyond 2 weeks of storage, the cells also became more fragile upon reconstitution as shown in Table 1. It is possible that a longer storage time at room temperature may further decrease the moisture content of the cells. As such, in this study, we confined our functional studies to 2 weeks in storage of cells at $25 \%$ residual moisture.

We examined the morphology of the reconstituted RBCs after a 2 week storage in HemSol ${ }^{\mathrm{TM}}$ at room temperature. The RBCs were then hydrated with plasma as described in the materials and methods and imaged under a microscope. Figure 1(a) show representative fields of the rehydrated RBCs stored in HemSol ${ }^{\mathrm{TM}}$ at room temperature for 2 weeks and control RBCs (Figure 1(b)) stored in Adsol ${ }^{\mathrm{TM}}$ at $4^{\circ} \mathrm{C}$ for 2 weeks, respectively. As seen in this figure, the majority of HemSol ${ }^{\mathrm{TM}}$ stored RBCs show typical smooth biconcave structures, while the majority of Adsol stored RBCs showed some crenation. These results demonstrate that the RBCs treated with HemSol $^{\mathrm{TM}}$ show less ultrastructural damages than RBCs stored at $4^{\circ} \mathrm{C}$ in Adsol ${ }^{\mathrm{TM}}$ for the same time period.

The main function of RBCs is to carry oxygen to tissues. The ability of RBCs to bind and release oxygen is influenced by many factors including $\mathrm{Hb}$ oxidative state and regulators of $\mathrm{Hb}$ oxygen binding [9]. The standard way to measure this property is to perform oxygen saturation studies. Figure 2 shows oxygen dissociation curves of RBCs stored desiccated in HemSol ${ }^{\mathrm{TM}}$ for 2 weeks. The reconstituted RBCs were compared to fresh

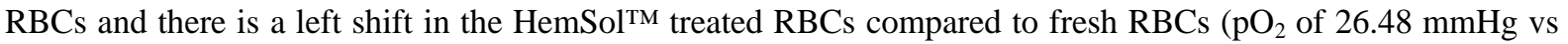
$17.10 \mathrm{mmHg}$ respectively). Both RBC preparations demonstrate a sigmoidal curve, indicating cooperative oxygen binding of hemoglobin. Additionally, both RBC preparations have similar $\mathrm{O}_{2}$ saturation concentrations, which indicates that most of the $\mathrm{Hb}$ in the desiccated/reconstituted RBCs is functional. Interestingly, the $\mathrm{pO}_{2}$ value of $17 \mathrm{mmHg}$ is comparable to RBCs stored in acid-citrate-dextrose-adenine for approximately 5 days at $4^{\circ} \mathrm{C}[15]$. 
To further examine $\mathrm{Hb}$ oxidation levels in desiccated cells, we measured the levels of methemoglobin (Met-Hb), which is an indication of oxidative stress associated with freeze-drying and desiccation [1] [13]. The heme group in Met-Hb has been oxidized from the oxygen carrying ferrous $\left(\mathrm{Fe}^{2+}\right)$ state to ferric $\left(\mathrm{Fe}^{3+}\right)$. Autoxidation of $\mathrm{Hb}$ to Met-Hb occurs naturally under normal physiologic conditions, accounting for around $1 \%$ of total $\mathrm{Hb}$ [3], but prolonged exposure of stored blood to atmospheric oxygen levels can increase Met-Hb levels. Desiccation of RBCs has been shown to increase the Met-Hb levels to 15\% - 50\% [1] [13]. Figure 3(b) shows that the Met-Hb was $4.5 \%$ in desiccated/reconstituted RBCs, compared to $0.9 \%$ in control RBCs. This data supports the results of Figure 2, which indicates that the majority of $\mathrm{Hb}$ in the desiccated/resuspended RBCs is functional. Although 4.5\% Met-Hb is lower than most desiccation procedures have reported, it is still higher than physiological concentrations ( $0 \%-3 \%)$ [3].

Since the data shown in Figure 2 and Figure 3(a) suggest that most of the Hb in desiccated/resuspended is capable of binding oxygen, we examined DPG levels in the HemSol ${ }^{\mathrm{TM}}$ treated RBCs to account for the left shift in the oxygen saturation experiment (Figure 2). This left shift is consistent with lower concentrations of 2,3diphosphoglycerate [16].

DPG is an important regulator of $\mathrm{Hb}$ oxygen binding. It binds deoxygenated $\mathrm{Hb}$, enhancing release of remaining bound oxygen. We measured the concentrations of DPG in the desiccated/reconstituted RBCs and those results shown in Figure 3(b) demonstrate there is a 20\% reduction in DPG concentrations in the HemSol ${ }^{\mathrm{TM}}$ treated cells. This is consistent with studies of RBC storage lesions, in which the steady depletion of DPG leads to a left shift in Hb oxygen binding properties.

\section{Conclusion}

Here, we describe a storage process for red cells that utilizes desiccation rather than refrigeration or freezing. The drying process does not involve freezing, thus ice-crystal damage and subsequent debris formation. It is clear that more work is needed to show HemSol ${ }^{\mathrm{TM}} \mathrm{RBC}$ desiccation as an alternative to regular RBC storage; however, to our knowledge, the present study shows that this process is superior to most other RBC desiccation procedures with respect to preserving $\mathrm{Hb}$ oxygen binding properties during desiccation and storage at room temperature for 2 weeks.

\section{Acknowledgements}

The authors wish to acknowledge the financial support of the Fort Detrick Technology Transfer Initiative, Ft. Detrick, MD and the award, administrative and contract support of TEDCO (the Maryland Technology Development Corporation, Columbia, MD). In addition, HeMemics appreciates the enthusiastic support thru a Cooperative Research and Development Agreement with US Army Institute of Surgical Research Fort Sam Houston, Texas.

\section{References}

[1] Kanias, T. and Acker, J.P. (2010) Biopreservation of Red Blood Cells-The Struggle with Hemoglobin Oxidation. FEBS Journal, 277, 343-356. http://dx.doi.org/10.1111/j.1742-4658.2009.07472.x

[2] Meryman, H.T. (1971) Osmotic Stress as a Mechanism of Freezing Injury. Cryobiology, 8, 489-500. http://dx.doi.org/10.1016/0011-2240(71)90040-X

[3] Mansouri, A. and Lurie, A.A. (1993) Methemoglobinemia. American Journal of Hematology, 42, 7-12. http://dx.doi.org/10.1002/ajh.2830420104

[4] Lynch, A.L., Dury, B.A.P., Guyader, C.P.E. and Slater, N.K.H. (2011) Sugars Comparable to Glutathione as Hemoglobin Oxidation Protectants During Vacuum Drying. Biopreservation and Biobanking, 9, 411-413. http://dx.doi.org/10.1089/bio.2011.0021

[5] Török, Z., Satpathy, G.R., Banerjee, M., Bali, R., Little, E., Novaes, R., Ly, H.V., Dwyre, D.M., Kheirolomoom, A., Tablin, F., Crowe, J.H. and Tsvetkova, N.M. (2005) Preservation of Trehalose-Loaded Red Blood Cells by Lyophilization. Cell Preservation Technology, 2, 96-111. http://dx.doi.org/10.1089/cpt.2005.3.96

[6] Weinstein, R., Sowemimo-Coker, S.O. and Goodrich, R.P. (1995) Survival of Lyophilized and Reconstituted Human Red Blood Cells in Vivo. Transfusion Clinique et Biologique, 2, 427-432. http://dx.doi.org/10.1016/S1246-7820(05)80067-9

[7] Han, Y., Quan, G.B., Liu, X.Z., Ma, E.P., Liu, A., Jin, P. and Cao, W. (2005) Improved Preservation of Human Red 
Blood Cells by Lyophilization. Cryobiology, 51, 152-164. http://dx.doi.org/10.1016/j.cryobiol.2005.06.002

[8] Ho, D., Schierts, J., Zimmerman, Z., Gadsden, I. and Bruttig, S. (2009) Comparison of Frozen versus Desiccated Reference Human Red Blood Cells for Hemagglutination Assays. Transfusion, 49, 2173-2180. http://dx.doi.org/10.1111/j.1537-2995.2009.02270.x

[9] Klein, H.G., Spahn, D.R. and Carson, J.L. (2007) Red Blood Cell Transfusion in Clinical Practice. The Lancet, 370, 415-426. http://dx.doi.org/10.1016/S0140-6736(07)61197-0

[10] Faivre, B., Menu, P., Labrude, P. and Vigneron, C. (1998) Hemoglobin Autoxidation/Oxidation Mechanisms and Methemoglobin Prevention or Reduction Processes in the Bloodstream Literature Review and Outline of Autoxidation Reaction. Artificial Cells, Blood Substitutes and Biotechnology, 26, 17-26. http://dx.doi.org/10.3109/10731199809118943

[11] Holme, S., Elfath, M.D. and Whitley, P. (1998) Evaluation of in Vivo and in Vitro Quality of Apheresis-Collected RBC Stored for 42 Days. Vox Sanguinis, 75, 212-217. http://dx.doi.org/10.1046/j.1423-0410.1998.7530212.x

[12] Hess, J.R. (2004) Red Cell Freezing and Its Impact on the Supply Chain. Transfusion Medicine, 14, 1-8. http://dx.doi.org/10.1111/j.0958-7578.2004.00472.x

[13] Kanias, T. and Acker, J.P. (2010) Mechanism of Hemoglobin-Induced Cellular Injury in Desiccated Red Blood Cells. Free Radical Biology and Medicine, 49, 539-547. http://dx.doi.org/10.1016/j.freeradbiomed.2010.04.024

[14] Loi, P., Iuso, D., Czernik, M., Zacchini, F. and Ptak, G. (2013) Towards Storage of Cells and Gametes in Dry Form. Trends in Biotechnology, 31, 688-695. http://dx.doi.org/10.1016/j.tibtech.2013.09.004

[15] Franklin Bunn, H., May, M.H., Kocholaty, W.F. and Shields, C.E. (1969) Hemoglobin Function in Stored Blood. Journal of Clinical Investigation, 48, 311-321. http://dx.doi.org/10.1172/JCI105987

[16] Benesch, R., Benesch, R.E. and Yu, C.I. (1968) Reciprocal Binding of Oxygen and Diphosphoglycerate by Human Hemoglobin. Proceedings of the National Academy of Sciences of the United States of America, 59, 526. http://dx.doi.org/10.1073/pnas.59.2.526 